\title{
Weißes Waldvögelein (Cephalanthera damasonium): \\ Orchidee des Jahres 2017
}

\author{
Angelika Baum, Heinz Baum \& Bernd Margenburg
}

\begin{abstract}
The white helleborine is orchid of the year 2017. This species is mostly found in forests and woods. We here outline its biology and morphology and elaborate on the etymology of its many names.
\end{abstract}

\section{Zusammenfassung}

Das Weiße Waldvögelein ist Orchidee des Jahres 2017. Sie kommt bevorzugt in Wäldern vor. Ihre Biologie und Morphologie werden beschrieben. Ihre vielen verschiedenen Namen werden erläutert.

\section{Einleitung}

Um auf die Problematik der Veränderung von Lebensräumen und ihrer Zerstörung aufmerksam zu machen, wird jährlich von den deutschen Arbeitskreisen Heimische Orchideen (AHO Deutschland) eine Orchidee des Jahres gewählt. Für das Jahr 2017 ist es das Weiße Waldvögelein (Cephalanthera damasonium). Sie ist eine der häufigsten Orchideen-Arten in Deutschland, die jedoch durch Intensivierung der Waldnutzung zunehmend gefährdet ist.

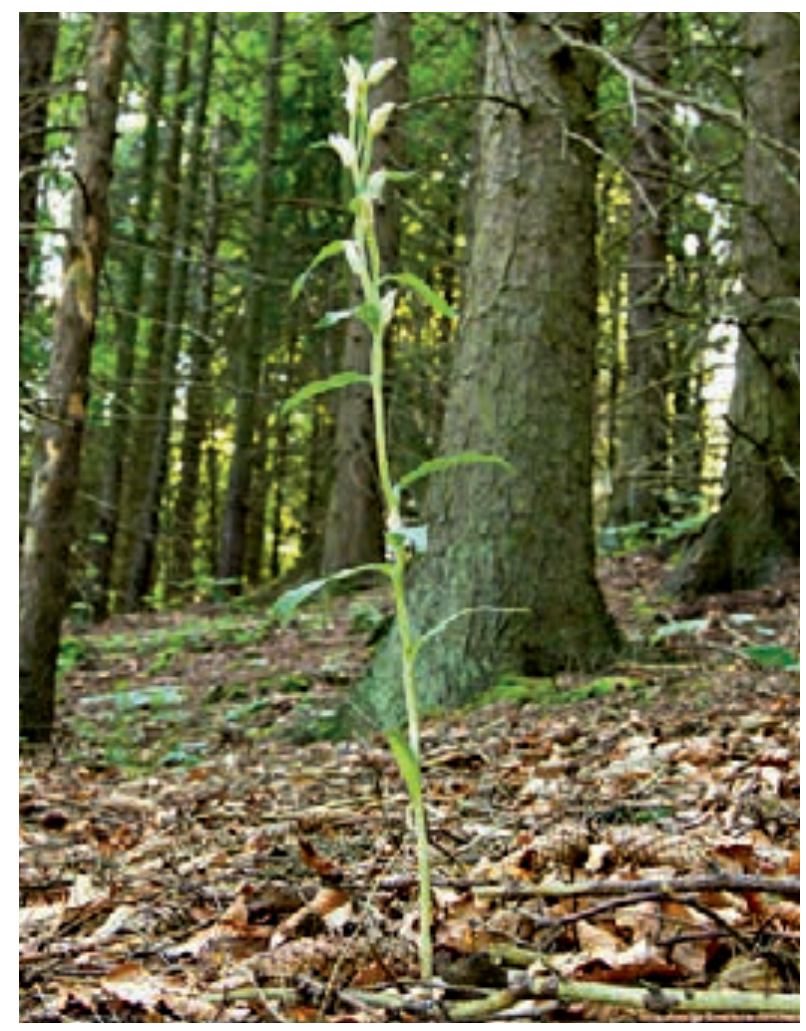

Abb. 1: Cephalanthera damasonium in einem Wald in der Eifel.

\section{Name}

Die Gattung Cephalanthera wurde im Jahr 1817 vom französischen Botaniker Louis Claude Marie Richard aufgestellt. Der Gattungsname setzt sich aus dem griechischen $\kappa \varepsilon \phi a \lambda \eta \dot{~(k e p h a l e ̄ ~=~}$ Kopf) und àvӨnoóc (anthērós = blühend) zusammen und weist darauf hin, dass die Anthere auf dem Säulchen wie ein Kopf aufsitzt.

Als Artbeiname wurde die Pflanzenbezeichnung aus den Zeiten vor Linné übernommen. An-

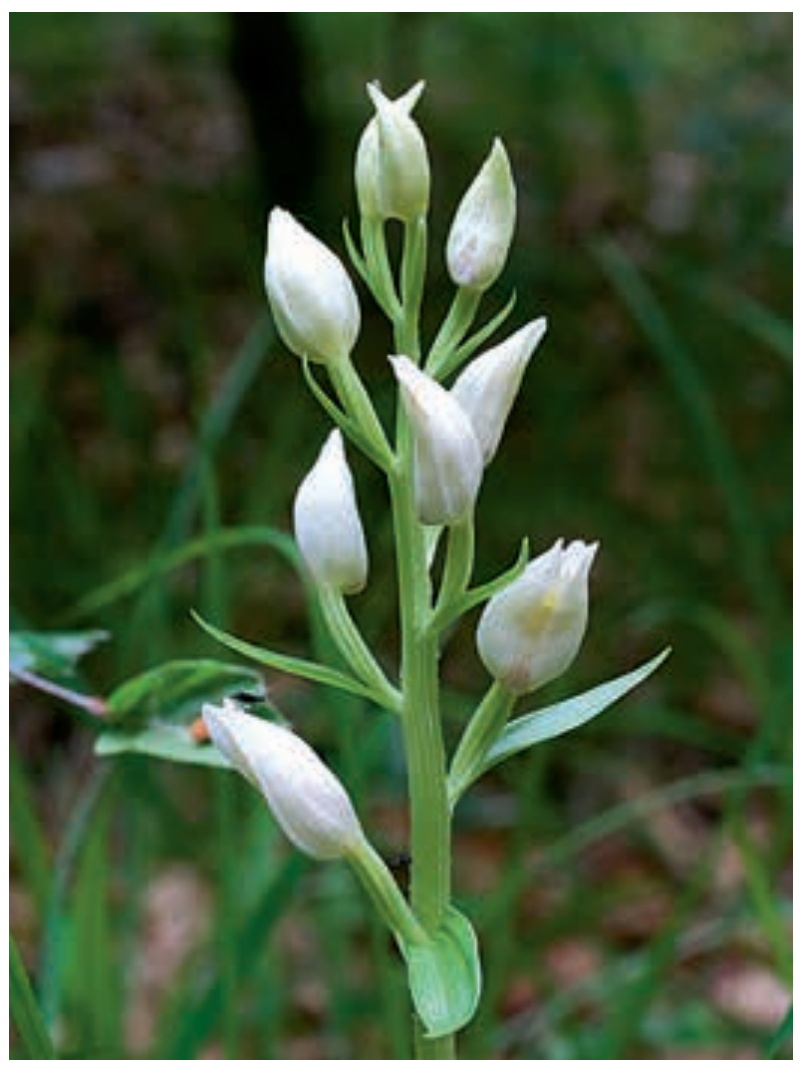

Abb. 2: Blütenstand von Cephalanthera damasonium. 


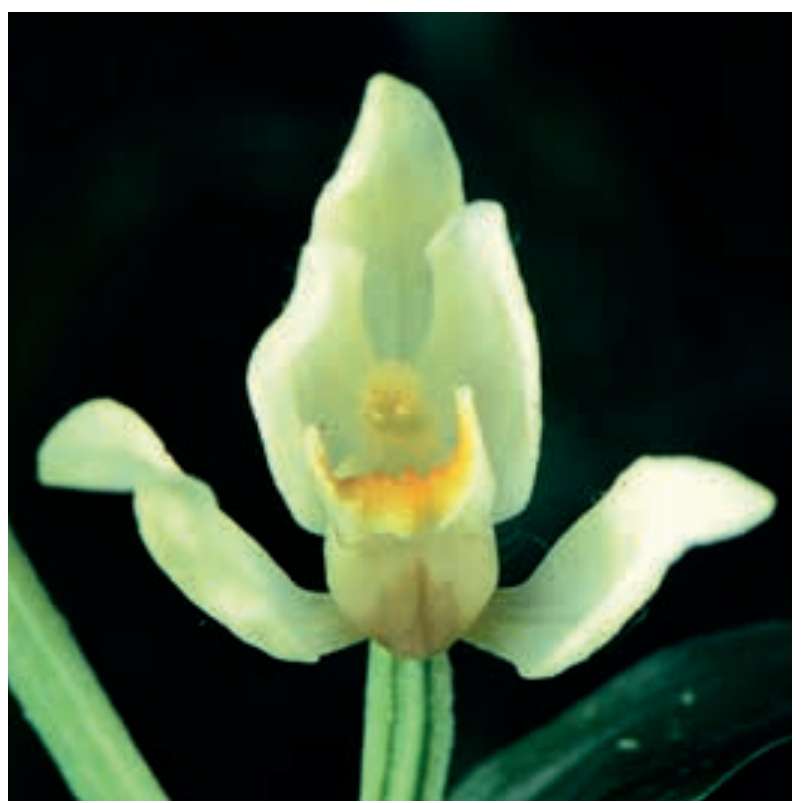

Abb. 3: Blüte von Cephalanthera damasonium.

fang des 17. Jahrhunderts wurde noch der Name Damasonium alpinum verwendet, der in Elleborine floribus albis (weiß) und floribus luteus (gelb, bleich) unterteilt wurde (BAUHIN $(1623,1651)$. Geht man noch weiter zurück, stößt man auf einen Artnamen aus den Zeiten von Dioskurides und Plinius (also aus dem 1. Jahrhundert n. Chr.). Pflanzen, deren Blätter denen des Wegerichs ähneln, wurden mit alisma, alcima, damasonion und lyron bezeichnet.

Weißes Waldvögelein ist der gebräuchlichste deutsche Name für diese Orchidee. In Bayern wird aber meist der Name Bleiches Waldvögelein verwendet. Diese Bezeichnung beschreibt die Blütenfarbe eigentlich besser. Weitere Volksnamen sind Kopfstendel, Kopfkölbchen, Rundbeutel, Kopfbeutel, Weißer Kuckuck, Falbes Waldvögelein (vom altgermanischen Adjektiv falo = fahl, grau, blass, weißlich oder blassgelb), Hennenkopf, Orant (lateinisch orans =der/die Betende) und Zymbelblume (Zịmbeln, kleine abgestimmte Becken, die entweder paarweise gegeneinander oder mit einem Schlägel einzeln angeschlagen werden).

Die eurasisch-nordamerikanisch verbreitete Gattung Cephalanthera umfasst je nach Autor 1418 Arten. Dazu zählen z. B. C. falcata im südlichen Japan, in Korea und Süd-China sowie C. kurdica im Südkaukasus, Irak, Iran und in der Türkei. Eine der drei mykotrophen Arten (C. austiniae) kommt in den westlichen USA und auf Vancouver Island vor. In Europa gibt es sieben Arten, nämlich C. cucullata, C. epipactoides, C. kotschyana, C. kurdica und die auch in Deutschland vorkommenden Arten C. rubra, C. longifolia und C. damasonium. Die erstgenannten vier Arten haben jeweils eng begrenzte Areale im Süden und Südosten Europas und Kleinasiens.

Das Weiße Waldvögelein wurde 1768 von Philip Miller als Serapias damasonium beschrieben. Mit der Gattungsbeschreibung von RICHARD 1817 wurde das Weiße Waldvögelein zu Cephalanthera pallens umkombiniert.

Der heute gültige wissenschaftliche Name C. damasonium wurde erst 1906 durch George Claridge Druce eingeführt. Im Laufe der Jahre gab es unzählige Namensänderungen bzw. Umkombinationen. Hier ein kleiner Auszug der in die Synonymie verwiesenen Namen:

- Serapias latifolia Miller

- Epipactis alba CRANTz

- Serapias grandiflora Oeder

- Serapias tota-alba Gilib.

- Epipactis lancifolia F. W. Schmidt

- Serapias alba (Crantz) SAlisb.

- Serapias lancifolia (F. W. Sснмidt) Roth

- Epipactis ochroleuca BAUMG.

- Cephalanthera pallens L. C. M. Richard

- Serapias ochroleuca (Baumg.) Steud.

- Cephalanthera lancifolia (F. W. Sснмidt) DumorT.

- Serapias pallens (Sw.) S. B. Jundz.

- Cephalanthera ochroleuca (BAumg.) Reichenbach

- Cephalanthera acuminata LEDEB.

- Cephalanthera alba (CRAnTz) SimonKai

- Cephalanthera latifolia JANCHEN

\section{Verbreitung und Lebensraum}

Die Verbreitung von Cephalanthera damasonium reicht in Europa nördlich bis nach Süd-Schwe- 


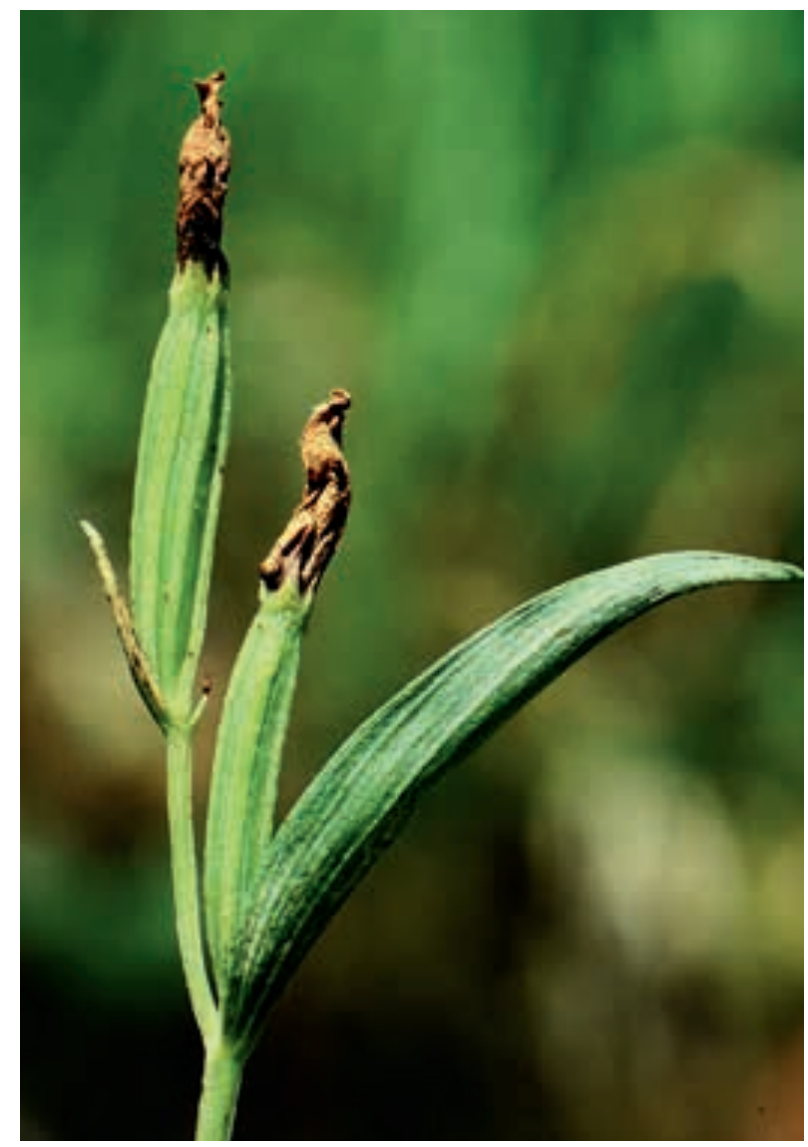

Abb. 4: Junge Früchte.

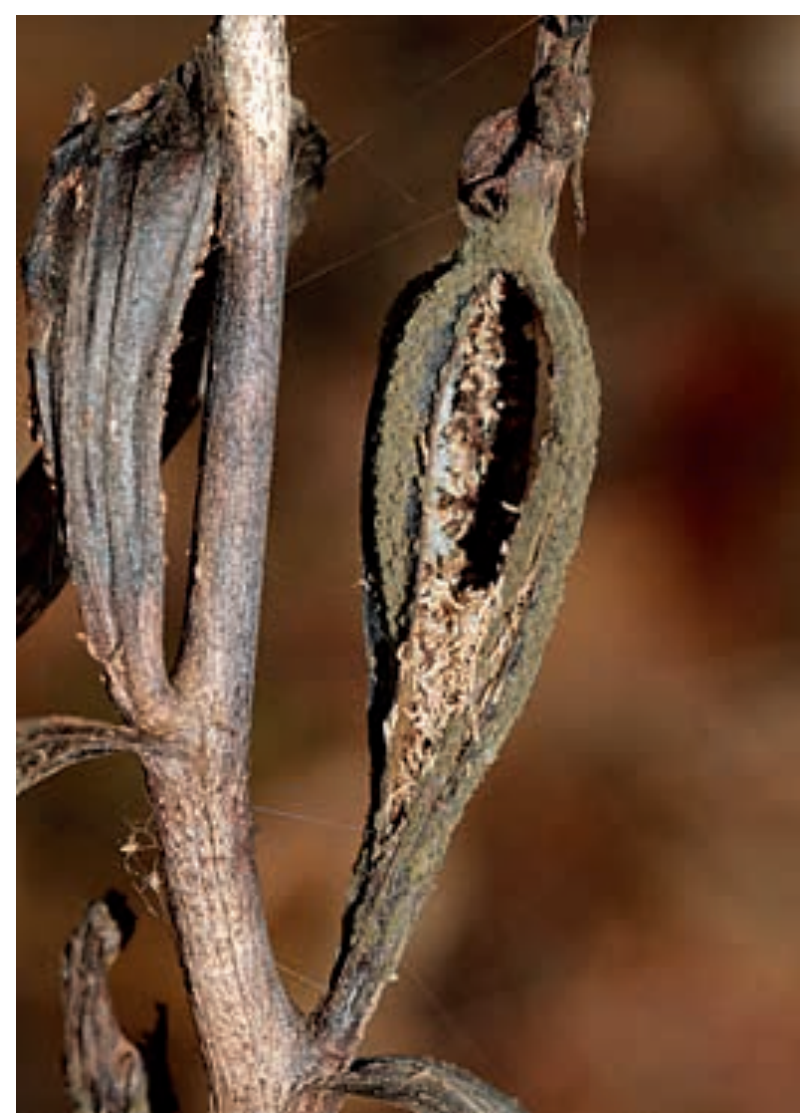

Abb. 5: Die Kapseln entlassen unzählige staubfeine Samen. den, südlich bis in den Mittelmeerraum und östlich bis in den kleinasiatischen Raum. In Deutschland meidet das Weiße Waldvögelein die Regionen mit weitgehend kalkfreien Böden. Daher ist es im norddeutschen Tiefland bis zum Rand der Mittelgebirgsschwelle, dem Bayerischen Wald, dem Schwarzwald und in Bayern zwischen Alpenvorland und Donau sehr selten oder es fehlt. Seine Höhenverbreitung endet bei 900 bis $1000 \mathrm{~m}$ ü. NN.

Das Weiße Waldvöglein kommt vor allem in Altbuchenbeständen und Buchen-Tannen-Wäldern vor. Teilweise findet man aber auch Pflanzen truppweise in Fichten- und Kiefernforsten sowie in Gebüschen trockenwarmer Standorte. Die Art steht gern schattig bis halbschattig und meidet zu dichten Unterwuchs. Besonders in jungen Fichtenmonokulturen mit einem Alter von etwa 15-20 Jahren kann es sich für kurze Zeit sehr stark ausbreiten. Funde im offenen Gelände sind Relikte von ehemaligem Wald oder Gebüschen.

Nach Haeupler \& Muer (2000) besiedelt Cephalanthera damasonium Buchen- und Buchenmischwälder sowie Trockenhang-Kalk-Buchenwälder auf flachgründigen Rendzina-Böden. Nach Ellenberg (1979) ist sie eine Schattenbis Tiefschattenpflanze, ein Mäßigwärmezeiger und ein Trocken- bis Frischezeiger. Sie findet sich als Schwachsäure- und Schwachbasenzeiger niemals auf stark sauren Böden und bevorzugt stickstoffarme bis mäßig stickstoffreiche Standorte. Ihre Verbreitung ist ozeanisch mit Schwergewicht im Westen einschließlich des westlichen Mitteleuropas.

\section{Morphologie und Biologie}

Cephalanthera damasonium ist ein sommergrüner Rhizomgeophyt mit einer oft verzweigten, waagerecht kriechenden Grundachse. Im Frühjahr entwickeln sich aus schlafenden Augen oberirdische Sprosse, die eine Wuchshöhe von 8-60 cm erreichen. Am Grund des grünen Stängels finden sich 1-3 dunkelbraune, schuppenartige Blätter. Nach oben folgen ziemlich gleichmäßig verteilt 3-6 grüne, meist etwas glänzende und mit Längsnerven versehene Blätter. Diese sind breit eiförmig 
bis eiförmig-lanzettlich und waagerecht abstehend bis aufwärts gerichtet.

Der Stängel ist kantig, langgestreckt, etwas hin und hergebogen. Der Blütenstand ist locker. Er erreicht eine Länge von ca. 6-22 cm und ist mit 2-20 leicht elfenbeinfarbenen, selten weißen, spornlosen Blüten besetzt. Die Tragblätter, vor allem im unteren Bereich des Blütenstands, sind deutlich länger als der Fruchtknoten plus Blüten. Der Fruchtknoten ist kantig, gedreht und nach oben gerichtet. Die Perigonblätter sind meist zusammen geneigt und öffnen sich selten ganz, daher resultiert der alte Name Rundbeutel. Die Kelchblätter sind eiförmig bis lanzettlich zugespitzt. Die Kronblätter sind etwas kürzer. Bei der unterteilten Lippe fällt die herzförmige Vorderlippe mit gelb bis orange gefärbten Längsleisten auf.

Hinter der dreieckigen Hinterlippe, die am Ende sackartig vertieft und dort dottergelb gefärbt ist, ist in der Blüte zunächst das Säulchen erkennbar. Die hellgelben Pollenkörner sind nicht zu festen Pollinien geformt, sodass sie sich aus den Antherenfächern lösen und mit der elliptischen Narbe in Kontakt kommen können. Die Pflanzen sind also selbstbestäubend. Deshalb ist es auch nicht nötig, dass sich die Blüten weit öffnen. Durch die großen, steil aufrecht stehenden Fruchtkapseln lässt sich das Weiße Waldvöglein auch nach der Blüte und sogar im vertrockneten Zustand noch gut bestimmen. Die Blütezeit erstreckt sich etwa von Mitte Mai bis Mitte Juni. Die Samenreife erfolgt von September bis Oktober.

Nicht selten tritt das Weiße Waldvögelein auch in kleinen Grüppchen auf, da es sich vegetativ aus dem stark verzweigten Rhizom vermehren kann. Bei zu starker Beschattung treten häufig blütenlose Exemplare auf. Die grünen Pflanzen beziehen etwa die Hälfte des Kohlenstoffbedarfs von den Wurzelpilzen.

Die Art ist wenig variabel. Sehr selten treten Monstrositäten oder Pflanzen mit hellgelb gefärbten Blüten auf. Selten sind auch chlorophyllarme bis ganz chlorophyllfreie Pflanzen und Albinos mit reinweißen Blüten zu finden.

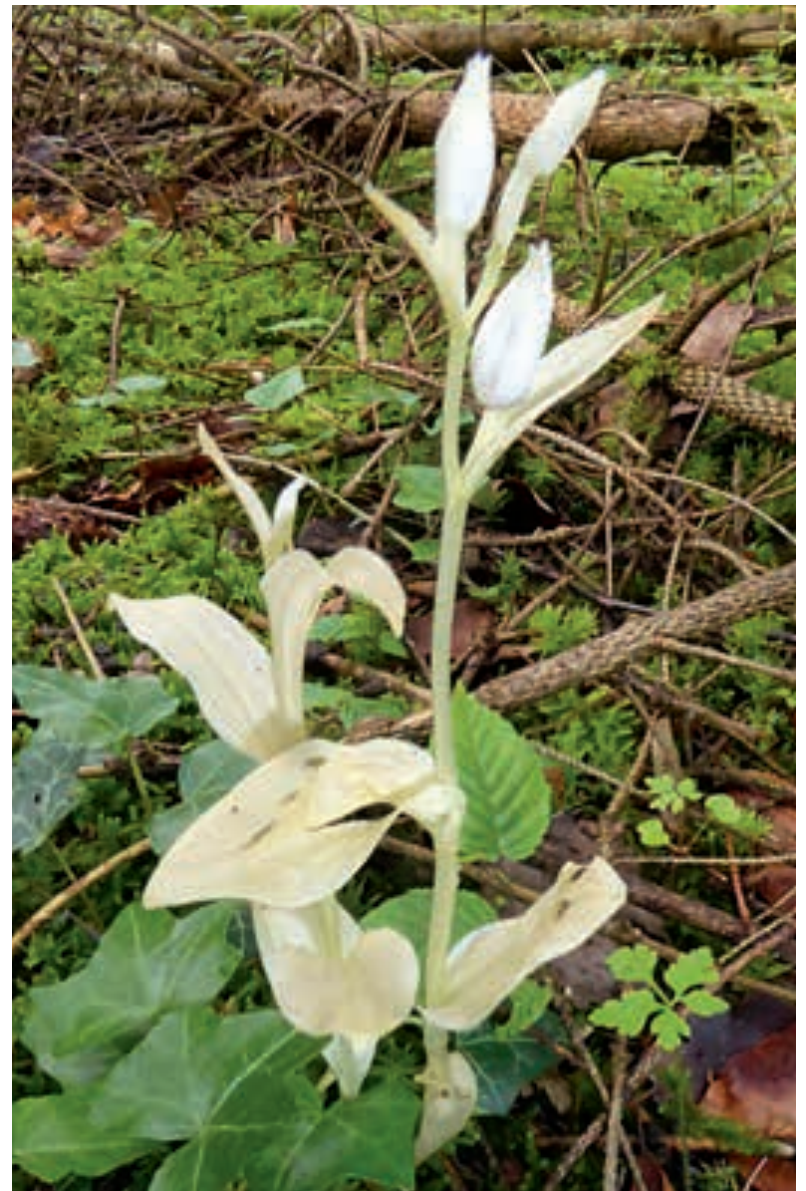

Abb. 6: Ein chloropyllfreies, bleiches Exemplar von Cephalanthera damasonium.

\section{Gefährdung}

In den meisten Bundesländern ist die Art nicht gefährdet. Auch in der Roten Liste Deutschlands wird sie nicht als gefährdet geführt. Trotz der noch stellenweise individuenreichen Vorkommen steht das Weiße Waldvöglein wie alle Orchideen-Arten unter strengem Schutz europäischer und nationaler Gesetze.

Aufmerksam verfolgt werden müssen jedoch Biotopverluste durch Abholzung und damit zu starker Auslichtung der Wälder oder durch Verbuschung. Schädigungen der Mykorrhiza durch forstliche Maßnahmen sind unbedingt zu vermeiden.

\section{Hybriden}

Hybriden mit den beiden anderen Cephalanthera-Arten sind sehr selten und viele Meldungen bleiben unbestätigt, obwohl die Arten sich ihre 
Standorte oft teilen. Folgende interspezifische Hybriden sind für Deutschland beschrieben:

- Cephalanthera $\times$ schulzei E. G. Camus (= C. damasonium $\times$ C. longifolia $)$

- Cephalanthera $\times$ mayeri (E. Mayer \& ZIMmerm.) A. Camus $(=C$. damasonium $\times C$. rubra)

Intergenerische Hybriden mit Epipactis geistern seit über 100 Jahren durch die Literatur, z. B. soll eine solche Hybride 1886 in Österreich gefunden worden sein. In der Literatur ist sie mehrfach als nicht glaubwürdig oder als in das Reich der Falschmeldungen gehörend eingestuft worden. Aktuell wird bei Heinrich et al. (2014) die Hybride Cephalopactis $\times$ speciosa (Settst.) Asch. \& Graebn. (= Cephalanthera damasonium $\times$ Epipactis atrorubens) genannt. Zumindest in Kultur wäre diese Hybride auch möglich. In der Natur bleibt sie sehr umstritten. Falls sie richtig bestimmt wurde, stellt sich die Frage, inwieweit sie anthropogenen Ursprungs ist. Andere Hybriden mit Dactylorhiza bzw. Gymnadenia wurden nie bestätigt:

\section{Dank}

Eva Schaller (Freudenburg), Jean Claessens (Geulle/NL) und Wilfried Kuhn (Wuppertal) danken wir für die freundlicherweise zur Verfügung gestellten Fotos.

\section{Literatur}

AHO Deutschland, Arbeitskreise Heimischer Orchideen (Hrsg.) 2005: Die Orchideen Deutschlands. - Uhlstädt-Kirchhasel.

AHO NRW, Arbeitskreis Heimische Orchideen NRW (Hrsg.) 2001: Die Orchideen Nordrhein-Westfalens. - Wuppertal.

Bauhin, C. 1623: Pinax Theatri Botanici. - Basel.

Bauhin, J. 1651: Historia plantarum vniuersalis, noua, et absolutissima, cum consensu 3: 516-517.

Camus E. G. 1921: × Cephalepipactis speciosa Cam. Berg. Cam. - Iconographie des Orchidées d'Europe et du Bassin méditerranéen. Planche 107. - Paris.

Claessens, J. \& Kleynen, J. 2011: The flower of the european orchid - form and function. - Voerendaal.

Ellenberg, H. 1979: Zeigerwerte der Gefäßpflanzen Mitteleuropas, 2. Aufl. - Scripta Geobot. 9.
Genaust, H. 1996: Cephalanthera-Etymologisches Wörterbuch der botanischen Pflanzennamen. - Hamburg.

Griebel, N. 2011: Die Orchideenhybriden Österreichs. - Ber. Arbeitskrs. Heim. Orchid. 28: 136.

Haeupler, H. \& Muer, T., 2000: Bildatlas der Farn- und Blütenpflanzen Deutschlands. - Stuttgart.

Haeupler, H., Jagel, A. \& Schumacher, W. 2003: Verbreitungsatlas der Farn- und Blütenpflanzen in Nordrhein-Westfalen. - Recklinghausen.

Heinrich, W., Voelckel, H., Dietrich, H., Feldmann, R., Geithner, A., Kögler, V., Rode, P. \& Westhus, W. 2014: Thüringens Orchideen. - Uhlstädt-Kirchhasel.

Korneck, D., Schnittler, M. \& Vollmer, I. 1996: Rote Liste der Farn- und Blütenpflanzen (Pteridophyta et Spermatophyta) Deutschlands. - Schriftenr. Vegetationskde. 28: 21-187.

Luer, C. A. 1975: Cephalanthera austinae (A. Gray) - The native orchids of the United States and Canada excluding Florida. Plate 14. - New York.

Marzell, H. 2000: Wörterbuch der deutschen Pflanzennamen. - Köln.

Raabe, U., Büscher, D., Fasel, P., Foerster, E., Götte, R., Haeupler, H., Jagel, A., Kaplan, K., Keil, P., Kulbrock, P., Loos, G. H., Neikes, N., Schumacher, W., Sumser, H. \& Vanberg, C. 2011: Rote Liste und Artenverzeichnis der Farn- und Blütenpflanzen - Pteridophyta et Spermatophyta - Nordrhein-Westfalen. - Recklinghausen.

Wisskirchen, R. \& Haeupler, H. 1998: Standardliste der Farn- und Blütenpflanzen Deutschlands. - Stuttgart.

\section{Internetseiten}

http://www.aho-bayern.de/taxa/ce_dama.html [06.01.2017].

http://www.orchids.de/galry/bw/Cephalanthera\%20damasonium.html [06.01.2017].

https://de.wikipedia.org/wiki/Wei\%C3\%9Fes_ Waldv\%C3\%B6glein [06.01.2017].

http://www.theplantlist.org/tpl/record/kew-36635 [06.01.2017].

https://de.wikipedia.org/wiki/Gew\%C3\%B6hnlicher_ Froschl\%C3\%B6ffel [06.01.2017].

http://www.floraweb.de/index.html [06.01.2017]. 